\title{
A NOTE ON SUMSETS OF SUBGROUPS IN $\mathbb{Z}_{p}^{*}$.
}

\author{
DERRICK HART
}

\begin{abstract}
Let $A$ be a multiplicative subgroup of $\mathbb{Z}_{p}^{*}$. Define the $k$-fold sumset of $A$ to be $k A=$ $\left\{x_{1}+\cdots+x_{k}: x_{i} \in A, 1 \leq i \leq k\right\}$. We show that $6 A \supseteq \mathbb{Z}_{p}^{*}$ for $|A|>p^{\frac{11}{23}+\epsilon}$. In addition, we extend a result of Shkredov to show that $|2 A| \gg|A|^{\frac{8}{5}-\epsilon}$ for $|A| \ll p^{\frac{5}{9}}$.
\end{abstract}

\section{INTRODUCTION}

For subsets $A_{1}, \ldots, A_{k}$ of a group define $A_{1}+\cdots+A_{k}=\left\{a_{1}+\cdots+a_{k}: a_{i} \in A_{i}, 1 \leq i \leq k\right\}$. In the case that all the subsets are equal we will denote the $k$-fold sumset of $A$ by $k A=\left\{x_{1}+\cdots+x_{k}\right.$ : $\left.x_{i} \in A, 1 \leq i \leq k\right\}$.

Let $A$ be a multiplicative subgroup of $\mathbb{Z}_{p}^{*}$. What is the smallest $\alpha>0$ such that $|A| \gg p^{\alpha}$ implies that $2 A$ contains $\mathbb{Z}_{p}^{*}$ ?

Conjecture 1. Let $|A|>p^{\frac{1}{2}+\epsilon}, \epsilon>0$ then $2 A$ contains $\mathbb{Z}_{p}^{*}$.

It is relatively simple, using exponential sum bounds, to show that if $|A|>p^{\frac{3}{4}}$ then $2 A \supseteq \mathbb{Z}_{p}^{*}$. Surprisingly, no improvement in the exponent has been made. An alternative approach would be to consider this conjecture from an inverse perspective. Let $|A|>p^{\frac{1}{2}+\epsilon}$; what is the smallest $k_{0}$ such that $k_{0} A$ contains $\mathbb{Z}_{p}^{*}$ ? A direct application of classical counting methods using standard exponential sum bounds does not seem to yield any answer to this question. For example, using the fact that $\max _{\lambda \neq 0}\left|\sum_{x \in A} e_{p}(x \lambda)\right| \leq \sqrt{p}$ one may show that if $|A|>p^{\frac{1}{2}+\frac{1}{2 k}}$ then $k A$ contains $\mathbb{Z}_{p}^{*}$.

Using combinatorial methods Glibichuk [1] gave the first answer to this question showing that $8 A \supseteq \mathbb{Z}_{p}^{*}$ for $|A| \geq 2 p^{\frac{1}{2}}$. Using an improved exponential sum bound, Schoen and Shkredov [5, Theorem 2.6] showed that $7 A \supseteq \mathbb{Z}_{p}^{*}$ for $|A|>p^{\frac{1}{2}}$. There was subsequent improvement to this result by Shkredov and Vyugin [7] followed by Schoen and Shkredov [6]. Recently, Shkredov [4] has shown that $6 A \supseteq \mathbb{Z}_{p}^{*}$ if $|A|>p^{\frac{55}{112}+\epsilon}=p^{.491 \ldots+\epsilon}$.

In this paper we elaborate on the methods in the above mentioned papers to show that $6 A \supseteq \mathbb{Z}_{p}^{*}$ if $|A|>p^{\frac{11}{23}+\epsilon}=p^{.478 \ldots+\epsilon}$. In addition, we extend a result of Shkredov ([4]) to show that $|2 A| \gg|A|^{\frac{8}{5}-\epsilon}$ for $|A| \ll p^{\frac{5}{9}}$.

\section{Statement of Main Results}

Let $A$ and $B$ be subsets of $\mathbb{Z}_{p}$. Given a set $A$ we will denote the indicator function of $A$ by $A(\cdot)$. Define the convolution of $A$ and $B$ by $(A * B)(z)=\sum_{x+y=z} A(x) B(y)=|A \cap(B+z)|$. 
The additive energy between $A$ and $B$ be given by,

$$
\begin{aligned}
E(A, B) & =|\{(x, y, z, w) \in A \times B \times A \times B: x+y=z+w\}| \\
& =\sum_{z}(A * B)^{2}(z)=\sum_{z}|A \cap(z-B)|^{2} \\
& =\sum_{z}(A *-A)(z)(B *-B)(z)=\sum_{z}\left|A_{z}\right|\left|B_{z}\right|,
\end{aligned}
$$

where here and throughout the paper we will let $C_{z}=C \cap(C+z)$ for any subset $C$ of $\mathbb{Z}_{p}$. In the case that $A=B$ we will write $E(A)=E(A, A)$. Similarly, we will denote the $r$ th additive energy of a subset $A$ by $E_{r}(A)=\sum_{s}\left|A_{s}\right|^{r}$.

One may also consider the additive energy in the frequency domain. Taking an exponential sum expansion, $E(A, B)=p^{-1} \sum_{s}\left|\sum_{x \in A} e_{p}(s x)\right|^{2}\left|\sum_{y \in A} e_{p}(s y)\right|^{2}$, where $e_{p}(x)=e^{\frac{2 \Pi i x}{p}}$. For a subset $A$ of $\mathbb{Z}_{p}$ we define $\Phi_{A}=\max _{\lambda \neq 0}\left|\sum_{x \in A} e_{p}(\lambda x)\right|$.

Heath-Brown and Konyagin employed Stepanov's method in order to give a bound on the additive energy of multiplicative subgroups of $\mathbb{Z}_{p}^{*}$.

Theorem 2 ([2]). Let $A$ be a multiplicative subgroup of $\mathbb{Z}_{p}^{*}$ with $|A| \ll p^{\frac{2}{3}}$. Then

$$
E(A) \ll|A|^{\frac{5}{2}} .
$$

In [4] Shkredov gave the following combinatorial lemma.

Lemma 3 ([4], Equation 1)). Let $A$ be a finite subset of an abelian group. Then

$$
\sum_{s} \frac{\left|A_{s}\right|^{2}}{\left|A+A_{s}\right|} \ll|A|^{-2} E_{3}(A) .
$$

Schoen and Shkredov ([5]) gave an estimate for $E_{3}(A)$.

Lemma 4 ([5], Lemma 3.3). Let $A$ be a multiplicative subgroup $A$ of $\mathbb{Z}_{p}^{*}$ with $|A| \ll p^{\frac{2}{3}}$. Then we have,

$$
E_{3}(A) \ll|A|^{3} \log (|A|) .
$$

Combining Lemma [4] and Lemma [5] and noting that $\left|A+A_{s}\right| \leq\left|(2 A)_{s}\right|$ gives the following lemma.

Lemma 5. Let $A$ be a multiplicative subgroup $A$ of $\mathbb{Z}_{p}^{*}$ with $|A| \ll p^{\frac{2}{3}}$. Then we have,

$$
\sum_{s} \frac{\left|A_{s}\right|^{2}}{\left|(2 A)_{s}\right|} \ll|A| \log (|A|) .
$$

Shkredov used this inequality in [4] to give the following estimate on the additive energy.

Theorem 6 ([4], Theorem 30). Let $A$ be a multiplicative subgroup of $\mathbb{Z}_{p}^{*}$ such that $|A| \ll p^{\frac{2}{3}}$. If $E(A) \ll|A|^{\frac{3}{2}} \sqrt{p} \log (|A|)$ then

$$
E(A) \ll|A|^{\frac{4}{3}}|2 A|^{\frac{2}{3}} \log (|A|) .
$$

In addition, using different methods he proved an energy estimate independent of the size of the sumset. 
Theorem 7 ([4], Theorem 34). Let $A$ be a multiplicative subgroup of $\mathbb{Z}_{p}^{*}$ such that $|A| \ll p^{\frac{2}{3}}$. Then

$$
E(A) \ll \max \left\{|A|^{\frac{22}{9}} \log (|A|),|A|^{3} p^{-\frac{1}{3}} \log ^{\frac{4}{3}}(|A|)\right\} .
$$

Combining Theorem 6 and Theorem 7 and applying the trivial estimate $|2 A| \geq|A|^{4} E^{-1}(A)$ gives the following sumset estimate.

Theorem 8. Let $A$ be a multiplicative subgroup of $\mathbb{Z}_{p}^{*}$ such that $|A| \ll p^{\frac{2}{3}}$. Then

$$
|2 A| \gg\left\{\begin{array}{lll}
|A|^{\frac{8}{5}} \log ^{-\frac{3}{5}}(|A|), & \text { if } & |A| \ll p^{\frac{9}{17}} \\
|A|^{\frac{14}{9}} \log ^{-1}(|A|), & \text { if } & |A| \ll p^{\frac{3}{5}} \log ^{\frac{3}{5}}(|A|) ; \\
|A| p^{\frac{1}{3}} \log ^{-\frac{4}{3}}(|A|), & \text { if } & |A| \gg p^{\frac{3}{5}} \log ^{\frac{3}{5}}(|A|) .
\end{array}\right.
$$

Here we give the following energy estimate.

Theorem 9. Let $A$ be a multiplicative subgroup of $\mathbb{Z}_{p}^{*}$ such that $|A| \ll p^{\frac{2}{3}}$. Then

$$
E(A) \ll \max \left\{|A|^{\frac{4}{3}}|2 A|^{\frac{2}{3}} \log ^{\frac{1}{2}}(|A|),|A||2 A|^{2} p^{-1} \log (|A|)\right\} .
$$

This allows us to improve Shkredov's sumset result in some ranges.

Theorem 10. Let $A$ be a multiplicative subgroup of $\mathbb{Z}_{p}^{*}$ such that $|A| \ll p^{\frac{2}{3}}$. Then

$$
|2 A| \gg\left\{\begin{array}{lll}
|A|^{\frac{8}{5}} \log ^{-\frac{3}{10}}(|A|), & \text { if } & |A| \ll p^{\frac{5}{9}} \log ^{-\frac{1}{18}}(|A|) ; \\
|A| p^{\frac{1}{3}} \log ^{-\frac{1}{3}}(|A|), & \text { if } & |A| \gg p^{\frac{5}{9}} \log ^{-\frac{1}{18}}(|A|) .
\end{array}\right.
$$

Using, Plancherel or orthogonality one can very quickly prove that for a multiplicative subgroups $A, \Phi_{A} \ll \sqrt{p}$ for $|A| \gg p^{\frac{1}{2}}$. This is only non-trivial when $|A|>p^{\frac{1}{2}}$. Shparlinski ([3]) improved this result in some ranges with the bound $\Phi_{A} \ll|A|^{\frac{7}{12}} p^{\frac{1}{6}}$ for $p^{\frac{2}{5}} \ll|A| \ll p^{\frac{4}{7}}$. Heath-Brown and Konyagin used the energy estimate of Theorem 2 to obtain the following improvement.

Theorem 11. Let $A$ be a multiplicative subgroup. Then,

$$
\Phi_{A} \ll\left\{\begin{array}{lll}
\sqrt{p}, & \text { if } & p^{\frac{2}{3}} \ll|A| \leq p ; \\
p^{\frac{1}{4}}|A|^{-\frac{1}{4}} E^{\frac{1}{4}}(A) \ll p^{\frac{1}{4}}|A|^{\frac{3}{8}}, & \text { if } & p^{\frac{1}{2}} \ll|A| \ll p^{\frac{2}{3}} . \\
p^{\frac{1}{8}} E^{\frac{1}{4}}(A) \ll p^{\frac{1}{8}}|A|^{\frac{5}{8}}, & \text { if } & p^{\frac{1}{3}} \ll|A| \ll p^{\frac{1}{2}} .
\end{array}\right.
$$

Using Shkredov's energy estimate, then one may improve this result in some ranges in the case that the sumset is small. Let $|A| \ll p^{\frac{1}{2}}$ then,

$$
\Phi_{A} \ll p^{\frac{1}{8}}|A|^{\frac{1}{3}}|2 A|^{\frac{1}{6}} \log ^{\frac{1}{4}} .
$$

Using the same methods used to prove Lemma 4 one may obtain $E_{3 / 2}(A) \ll|A|^{\frac{9}{4}}$. In the case that the sumset is small we are able to significantly improve this bound.

Lemma 12. Let $A$ be a multiplicative subgroup with $|A| \ll p^{\frac{1}{2}}$. Then

$$
E_{3 / 2}(A) \ll|A|^{\frac{1}{2}}|2 A| \log ^{\frac{7}{4}}|A| .
$$

This Lemma allows us to obtain the following exponential sum bound which is an improvement of the result of Shkredov as long as $|2 A| \ll|A|^{\frac{7}{4}}$. 
Lemma 13. Let $A$ be a multiplicative subgroup with $|A| \ll p^{\frac{1}{2}}$. Then

$$
\Phi_{A} \ll p^{\frac{1}{8}}|A|^{-\frac{1}{8}}|2 A|^{\frac{1}{4}} E^{\frac{1}{8}}(|A|) \log \frac{7}{16}(|A|) .
$$

In particular, applying Theorem 9 we have

$$
\Phi_{A} \ll p^{\frac{1}{8}}|A|^{\frac{1}{24}}|2 A|^{\frac{1}{3}} \log ^{\frac{5}{8}}(|A|) .
$$

With Lemma 13 in tow, we may now prove our main result.

Theorem 14. Let $A$ be a multiplicative subgroup of $\mathbb{Z}_{p}^{*}$ with $|A| \gg p^{\frac{11}{23}} \log ^{\frac{15}{23}}(|A|)$. Then

$$
6 A \supseteq \mathbb{Z}_{p}^{*}
$$

Proof. Fix $a$ in $\mathbb{Z}_{p}^{*}$. We my assume that $|A| \ll p^{\frac{1}{2}}$ as the result is already known in the range $|A| \gg p^{\frac{1}{2}}$.

Let $N$ be the number of solutions to the equation,

$$
x_{1}+x_{2}+y_{1}+y_{2}=a y_{3},
$$

with $x_{1}, x_{2} \in 2 A$ and $y_{1}, y_{2} \cdot y_{3} \in A$.

Taking an exponential sum expansion,

$$
N=\frac{|2 A|^{2}|A|^{3}}{p}+\frac{1}{p} \sum_{\lambda \neq 0}\left(\sum_{x \in 2 A} e_{p}(\lambda x)\right)^{2}\left(\sum_{y \in A} e_{p}(\lambda y)\right)^{2}\left(\sum_{z \in A} e_{p}(-\lambda z a)\right),
$$

which by Plancherel implies that we have that $N>0$ as long as, $|2 A \| A|^{3}>p \Phi_{A}^{3}$.

Applying Theorem gives the condition,

$$
|2 A||A|^{3} \gg p^{\frac{11}{8}}|2 A||A|^{\frac{1}{8}} \log ^{\frac{15}{8}}(|A|),
$$

which in turn gives the condition,

$$
|A| \gg p^{\frac{11}{23}} \log \frac{15}{23}(|A|) .
$$

\section{A Few Preliminary Lemmas}

We begin with a lemma of Shkredov and Vyugin [7, Corollary 5.1] which is a generalization of a result of Heath-Brown and Konyagin [2]. We say that a subset $S \neq\{0\}$ is $A$-invariant if $S A=\{s a: s \in S, a \in A\}=S$, that is $S$ is a union of cosets of $A$ and possibly $\{0\}$.

Lemma 15. (Shkredov and Vyugin [7, Corollary 5.1]) Let $A$ be a multiplicative subgroup of $\mathbb{Z}_{p}$ and $S_{1}, S_{2}, S_{3}$ be $A$-invariant sets such that $\left|S_{1} \backslash\{0\}\right|\left|S_{2} \backslash\{0\}\right|\left|S_{3} \backslash\{0\}\right| \ll \min \left\{|A|^{5}, p^{3}|A|^{-1}\right\}$. Then

$$
\sum_{z \in S_{3}}\left(S_{1} * S_{2}\right)(z) \ll|A|^{-1 / 3}\left(\left|S_{1}\right|\left|S_{2}\right|\left|S_{3}\right|\right)^{2 / 3}
$$

Remark 3.1. The above lemma has been modified slightly from its original form in order to allow $S_{1}, S_{2}, S_{3}$ contain the zero element. One may check that the additional terms in $\sum_{z \in S_{3}}\left(S_{1} * S_{2}\right)(z)$ allowing $S_{1}, S_{2}$, and to contain the zero element only affect the implied constant. 
We can now give slight generalizations of several results of Schoen and Shkredov ([5], [6]).

Lemma 16. Let $k \gg 1$ and $S_{1}, S_{2}$ be A-invariant sets and let $M$ be any $A$-invariant subset of the set $\left\{z:\left(S_{1} * S_{2}\right)(z) \geq k\right\}$. If $\left|S_{1}\right|\left|S_{2}\right||M||A| \ll \min \left\{|A|^{6}, p^{3}\right\}$ then for $r \geq 1, r \neq 3$,

$$
\sum_{z \in M}\left(S_{1} * S_{2}\right)^{r}(z) \ll\left|S_{1}\right|^{2}\left|S_{2}\right|^{2}|A|^{-1} k^{r-3},
$$

and

$$
\sum_{z \in M}\left(S_{1} * S_{2}\right)^{3}(z) \ll\left|S_{1}\right|^{2}\left|S_{2}\right|^{2}|A|^{-1} \log \left(\left|S_{1}\right|^{2}\left|S_{2}\right|^{2}|A|^{-2} k^{-3}\right)
$$

Proof. Let $l_{i}=\left(S_{1} * S_{2}\right)\left(z_{i}\right), z_{i} \neq 0$ where $l_{1} \geq l_{2} \geq \ldots$ are arranged in decreasing order. For each $z$ in the coset $a A=\left\{a a^{\prime}: a^{\prime} \in A\right\}, a \in \mathbb{Z}_{p}$ note that $\left(S_{1} * S_{2}\right)(z)=\left(S_{1} * S_{2}\right)(a)$. By the coset $a_{i} A$ we will mean the coset on which $l_{i}=\left(S_{1} * S_{2}\right)\left(a_{i}\right)$. Let $M$ be any $A$-invariant subset of the set $\left\{z:\left(S_{1} * S_{2}\right)(z) \geq k\right\}$ and $M_{i}=\cup_{j=1}^{i} a_{j} A \subseteq M$. From Lemma 15 we have that

$$
l_{i}|A| i \leq \sum_{j=1}^{i}|A| l_{j} \leq \sum_{z \in M_{i}}\left(S_{1} * S_{2}\right)(z) \ll i^{2 / 3}|A|^{\frac{1}{3}}\left|S_{1}\right|^{\frac{2}{3}}\left|S_{2}\right|^{\frac{2}{3}},
$$

as long as $i|A|\left|S_{1}\right|\left|S_{2}\right| \ll|M|\left|S_{1}\right|\left|S_{2}\right| \ll \min \left\{|A|^{5}, p^{3}|A|^{-1}\right\}$. Now,

$$
\begin{aligned}
\sum_{z \in M}\left(S_{1} * S_{2}\right)^{r}(z) & \leq|A| \sum_{i \ll\left|S_{1}\right|^{3}\left|S_{2}\right|^{3}|A|^{-2} k^{-3}} l_{i}^{r} \\
& \ll|A| \sum_{i \ll\left|S_{1}\right|^{2}\left|S_{2}\right|^{2}|A|^{-2} k^{-3}}\left(i^{-\frac{1}{3}}|A|^{-\frac{2}{3}}\left|S_{1}\right|^{\frac{2}{3}}\left|S_{2}\right|^{\frac{2}{3}}\right)^{r} .
\end{aligned}
$$

\section{Additive Energy Bound: Proof of Theorem 9}

We may assume that $E(A) \gg \max \left\{|A|^{\frac{4}{3}}|2 A|^{\frac{2}{3}} \log ^{\frac{1}{2}}(|A|),|A||2 A|^{2} p^{-1} \log (|A|)\right\}$. Combining this with the energy estimate from Theorem 2 we may also assume that

$$
|2 A| \ll \max \left\{|A|^{\frac{7}{4}} \log ^{-\frac{3}{4}}(|A|),|A|^{\frac{3}{4}} p^{\frac{1}{2}} \log ^{-\frac{1}{2}}(|A|)\right\} .
$$

Write,

$$
E(A)=\sum_{s}\left|A_{s}\right|^{2} \ll \sum_{s \in M_{1}}\left|A_{s}\right|^{2},
$$

where $M_{1}=\left\{s:\left|A_{s}\right| \gg k_{1}:=|A|^{-2} E(A)\right\}$. Note that we have the trivial estimate $\left|M_{1}\right| \ll$ $|A|^{2} k_{1}^{-1}=|A|^{4} E^{-1}(|A|)$. Now by Lemma 5 we have,

$$
E(A)=\sum_{s}\left|A_{s}\right|^{2} \ll \frac{E(A)}{|A| \log (A)} \sum_{s \in M_{2}^{c}} \frac{\left|A_{s}\right|^{2}}{\left|(2 A)_{s}\right|}+\sum_{s \in M_{2}}\left|A_{s}\right|^{2} \ll \sum_{s \in M_{2}}\left|A_{s}\right|^{2},
$$

where $M_{2}=\left\{s: s \in M_{1},\left|(2 A)_{s}\right| \gg k_{2}:=|A|^{-1} \log ^{-1}(|A|) E(A)\right\}$.

By Lemma 15 we have that $k_{2}\left|M_{2}\right| \ll|A|^{-\frac{1}{3}}|2 A|^{\frac{4}{3}}\left|M_{2}\right|^{\frac{2}{3}}$ yielding $\left|M_{2}\right| \ll|2 A|^{4}|A|^{-1} k_{2}^{-3}$ as long as $|2 A|^{2}\left|M_{2}\right| \ll \min \left\{|A|^{5}, p^{3}|A|^{-1}\right\}$. In order to see that first condition is satisfied, one may note that $\left|M_{2}\right| \ll\left|M_{1}\right|$ combined with our assumptions on the size of energy and sumset. To show that 
$|2 A|^{2}\left|M_{2}\right| \ll p^{3}|A|^{-1}$ we use an exponential sum expansion,

$$
\left|M_{2}\right| k_{2} \ll \sum_{s \in M}\left|(2 A)_{s}\right| \ll \frac{1}{p} \sum_{m}\left|\sum_{x \in 2 A} e_{p}(x m)\right|^{2}\left(\sum_{x \in M_{2}} e_{p}(x m)\right),
$$

followed by applying the bound $\max _{m \neq 0}\left|\sum_{x \in M_{2}} e_{p}(x m)\right| \ll p^{\frac{1}{2}}\left|M_{2}\right|^{\frac{1}{2}}|A|^{-\frac{1}{2}}$ to give,

$$
\left|M_{2}\right| k_{2} \ll \max \left\{p^{-1}|2 A|^{2}\left|M_{2}\right|, p^{\frac{1}{2}}|2 A|\left|M_{2}\right|^{\frac{1}{2}}|A|^{-\frac{1}{2}}\right\} .
$$

If the first of these two bounds hold then we have $E(A) \ll|A||2 A|^{2} p^{-1} \log (|A|)$. We may then assume that $\left|M_{2}\right| \ll p|2 A|^{2}|A|^{-1} k_{2}^{-2}$ which implies that $|2 A|^{2}\left|M_{2}\right| \ll p|2 A|^{4}|A| \log ^{2}(|A|) E^{-2}(A) \ll$ $p^{3}|A|^{-1}$.

Therefore, for $|A| \ll p^{\frac{2}{3}}$, we have that $\left|M_{2}\right| \ll|2 A|^{4}|A|^{-1} k_{2}^{-3}$. Using this fact we may again reduce the number of terms,

$$
E(A)=\sum_{s}\left|A_{s}\right|^{2} \ll k_{3}^{2}\left|M_{2}\right|+\sum_{s \in M_{3}}\left|A_{s}\right|^{2} \ll \sum_{s \in M_{3}}\left|A_{s}\right|^{2},
$$

where $M_{3}=\left\{s: s \in M_{2},\left|A_{s}\right| \gg k_{3}:=|2 A|^{-2}|A|^{-1} \log ^{-\frac{3}{2}}(|A|) E^{2}(A)\right\}$.

Finally, applying Lemma 16 we have,

$$
E(A) \ll|A|^{4}|2 A|^{2} \log ^{\frac{3}{2}}(|A|) E^{-2}(|A|),
$$

as long as $|A|^{2}\left|M_{3}\right| \ll|2 A|^{2}\left|M_{2}\right| \ll \min \left\{|A|^{5}, p^{3}|A|^{-1}\right\}$.

\section{5. $E_{3 / 2}(A)$ : Proof of Lemma 12}

Let $l_{i}=\left|A_{z_{i}}\right|, z_{i} \neq 0$ where $l_{1} \geq l_{2} \geq \ldots$ are arranged in decreasing order. For each $z$ in the coset $a A=\left\{a a^{\prime}: a^{\prime} \in A\right\}, a \in \mathbb{Z}_{p}$ note that $\left|A_{z}\right|=\left|A_{a}\right|$. By the coset $a_{i} A$ we will mean the coset on which $l_{i}=\left|A_{a_{i}}\right|$. Let $M$ be any $A$-invariant subset of the set $\left\{z:\left|A_{z}\right| \geq k\right\}$ and $M_{i}=\cup_{j=1}^{i} a_{j} A \subseteq M$. Set $k=|2 A|^{2}|A|^{-3}$.

We have that

$$
l_{i}|A| i \leq \sum_{j=1}^{i}|A| l_{j} \leq \sum_{z \in M_{i}}\left|A_{z}\right|
$$

Now

$$
\sum_{z \in M_{i}}\left|A_{z}\right|=\sum_{z \in M_{i}} \frac{\left|A_{z}\right|}{\left|(2 A)_{z}\right|^{\frac{1}{2}}}\left|(2 A)_{z}\right|^{\frac{1}{2}} \leq\left(\sum_{z} \frac{\left|A_{z}\right|^{2}}{\left|2 A_{z}\right|}\right)^{\frac{1}{2}}\left(\sum_{z \in M_{i}}\left|2 A_{z}\right|\right)^{\frac{1}{2}} .
$$

Therefore, by Lemma 5 we have that

$$
l_{i}^{2}|A|^{2} i^{2} \ll|A| \log (|A|) \sum_{z \in M_{i}}\left|2 A_{z}\right|,
$$

Noting that $\left|M_{i}\right| \ll|A|^{2} k^{-1}$ we have $\left|M_{i}\right||2 A|^{2} \ll|A|^{5}$. Therefore we can apply Lemma 15 to give,

$$
l_{i}^{2}|A|^{2} i^{2} \ll|2 A|^{\frac{4}{3}} i^{\frac{2}{3}}|A|^{\frac{4}{3}} \log |A| .
$$

Therefore

$$
l_{i} \ll|2 A|^{\frac{2}{3}} i^{-\frac{2}{3}}|A|^{-\frac{1}{3}} \log ^{\frac{1}{2}}|A|,
$$


for $i \ll|A-A||A|^{-1} \leq|A|$.

Now,

$$
\begin{aligned}
\sum_{z}\left|A_{z}\right|^{\frac{3}{2}} & \ll k^{\frac{1}{2}}|A|^{2}+|A| \sum_{i \ll|A|}\left|l_{i}\right|^{\frac{3}{2}} \\
& \ll k^{\frac{1}{2}}|A|^{2}+|A|^{\frac{1}{2}}|2 A| \log ^{\frac{7}{4}}(|A|),
\end{aligned}
$$

giving the desired result.

\section{Exponential Sum Bound: Proof of Lemma 13}

We begin by expanding the sum below and performing a basic substitution,

$$
\begin{aligned}
|A|\left|\sum_{x \in A} e_{p}(\lambda x)\right|^{2} & =\sum_{y \in A}\left|\sum_{x \in A} e_{p}(\lambda y x)\right|^{2} \\
& =\sum_{x_{1}, x_{2} \in A} \sum_{y \in A} e_{p}\left(\lambda y\left(x_{1}-x_{2}\right)\right)=\sum_{s}\left|A_{s}\right| \sum_{y \in A} e_{p}(\lambda y s) .
\end{aligned}
$$

Now we may take absolute values and estimate from above,

$$
|A| \Phi_{A}^{2} \leq \sum_{s}\left|A_{s}\right|\left|\sum_{y \in A} e_{p}(\lambda y s)\right|
$$

Applying Holder we have,

$$
|A| \Phi_{A}^{2} \ll\left(\sum_{s}\left|A_{s}\right|^{\frac{4}{3}}\right)^{\frac{3}{4}}\left(\sum_{s}\left|\sum_{y \in A} e_{p}(\lambda y s)\right|^{4}\right)^{\frac{1}{4}}
$$

which by Plancherel gives,

$$
|A| \Phi_{A}^{2} \ll\left(\sum_{s}\left|A_{s}\right|^{\frac{4}{3}}\right)^{\frac{3}{4}} p^{\frac{1}{4}} E^{\frac{1}{4}}(A) .
$$

Now again applying Holder,

$$
\sum_{s}\left|A_{s}\right|^{\frac{4}{3}}=\sum_{s}\left|A_{s}\right|\left|A_{s}\right|^{\frac{1}{3}} \ll\left(\sum_{s}\left|A_{s}\right|^{\frac{3}{2}}\right)^{\frac{2}{3}}|A|^{\frac{2}{3}},
$$

and applying Lemma 12,

$$
\sum_{s}\left|A_{s}\right|^{\frac{4}{3}} \ll|A|^{\frac{2}{3}}\left(|A|^{\frac{1}{2}}|2 A| \log ^{\frac{7}{4}}(|A|)\right)^{\frac{2}{3}} \ll|A||2 A|^{\frac{2}{3}} \log ^{\frac{7}{6}}(|A|) .
$$

Putting this estimate into (1) gives the stated result. 


\section{REFERENCES}

[1] A. A. Glibichuk, Combinational properties of sets of residues modulo a prime and the Erdös-Graham problem, Mat. Zametki 79, no. 3, (2006), 384-395. Translated in Math. Notes 79, no. 3, (2006), 356-365. 1

[2] D. R. Heath-Brown and S. V. Konyagin,New bounds for Gauss sums derived from kth powers, and for Heilbronn's exponential sum, Q. J. Math. 51 (2000), no. 2, 221-235. 2, 4

[3] I. E. Shparlinski, On Bounds of Gaussian Sums, Mat. Zametki, 50 (1991),122130. 3

[4] I. D. Shkredov, Some new inequalities in additive combinatorics, preprint. 1, 2, 3

[5] T. Schoen and I. D. Shkredov, On a question of Cochrane and Pinner concerning multiplicative subgroups, arXiv:1008.0723v2, May 27, 2011, 1-10. 1, 2, 5

[6] _ Higher moments of convolutions, arXiv:1110.2986v1, Oct. 13, 2011, 1-35. 1, 5

[7] I. D. Shkredov and I. V. Vyugin, On additive shifts of multiplicative subgroups, arXiv:1102.1172v1, Feb. 6, 2011, 1-18. 1, 4

Department of Mathematics, Kansas State University, Manhattan, KS 66506

E-mail address: dnhart@math.ksu.edu 